\title{
Factors influencing the quality of life perception in patients with type 2 diabetes mellitus
}

\author{
This article was published in the following Dove Press journal: \\ Patient Preference and Adherence \\ 8 December 2016 \\ Number of times this article has been viewed
}

\section{Romulus Timar' \\ Iulian Velea ${ }^{2}$ \\ Bogdan Timar ${ }^{3}$ \\ Diana Lungeanu ${ }^{3}$ \\ Cristian Oancea ${ }^{4}$ \\ Deiana Roman ${ }^{5}$ \\ Octavian Mazilu ${ }^{6}$}

'Second Department of Internal Medicine, ${ }^{2}$ Department of Pediatrics, ${ }^{3}$ Department of Functional

Sciences, ${ }^{4}$ Department of Infectious Diseases, ${ }^{5}$ Faculty of General

Medicine, "Victor Babes" University of Medicine and Pharmacy, ${ }^{6}$ First

Department of Surgery, "Victor Babes" University of Medicine and Pharmacy, Timisoara, Romania
Correspondence: Iulian Velea Department of Pediatrics, "Victor Babes" University of Medicine and Pharmacy, 2 Eftimie Murgu, Timisoara 300076, Romania

Tel +40 256744616190

Fax +40 256462856

Email ivelea56@yahoo.com
Purpose: To evaluate the impact of several factors on the patient's perception on quality of life in a group of patients with type 2 diabetes mellitus (T2DM).

Patients and methods: In this cross-sectional study, 198 patients with T2DM were enrolled according to a consecutive-case population-based study design. In all participants, the perception on the quality of life was measured using the quality of life index - diabetes version III proposed by Ferrans and Powers. We evaluated the impact of several anthropometric and diabetes-related (ie, diabetes history and quality of glycemic control) factors on the patient's perception on the quality of life.

Results: The presence of diabetes complications was associated with a decreased quality of life: retinopathy ( 1 vs 5 points; $P<0.001$ ), chronic kidney disease ( -1 vs 5 points; $P<0.001$ ), and neuropathy ( -1 vs 5 points; $P<0.001$ ). A significant reverse correlation was found between the patient's quality of life and depression's severity (Spearman's $r=-0.345$; $P<0.001$ ) and body mass index (Spearman's $r=-0.158 ; P=0.026$ ). A positive association between the quality of life and the quality of diabetes-related self-care activities was found (Spearman's $r=0.338 ; P<0.001$ ). No significant association was found between the patient's quality of life and the quality of glycemic control, diabetes duration, age, gender, or smoking status.

Conclusion: To improve the patient's quality of life, special care should be given to the modifiable diabetes-related factors: the prevention and treatment of diabetes complications, treatment of depression, and weight loss in obese and overweight patients.

Keywords: quality of life, type 2 diabetes, diabetes complications, diabetes-related self-care activities

\section{Introduction}

The marked increase in life expectancy has made it obvious that there is a need for other health-related measures other than life expectancy and cause of death, especially regarding the grade of lived years. ${ }^{1}$ An individual's existence in all its aspects can be summed up using the concept known as "quality of life" (QoL). Narrowing the specter and referring to the health domain of a person's existence, the term "health-related quality of life (HRQoL)" is used as a subset, thus differentiating it from other QoL aspects. Since health embodies multidimensional domains such as physical, emotional, mental, and social functioning, they are also taken into account when speaking of HRQoL. ${ }^{2,3}$ In order to measure the differences in the patient's QoL at a point in time or during a certain period of time, interviewer or self-administered questionnaires are a useful tool. ${ }^{4}$

Diabetes mellitus (DM) is a group of metabolic diseases consisting of defects in what the secretion and/or action of insulin is regarded, leading to the main manifestation, 
hyperglycemia. ${ }^{5}$ It represents a globally rising health issue, the number of people suffering from this chronic disease last being estimated in 2016 by the World Health Organization at 422 million. ${ }^{6}$ The estimated number of affected individuals by the year 2030 is expected to double. ${ }^{7}$

Chronic hyperglycemia as found in diabetes is associated with organ dysfunction, damage or even failure, and diabetes-specific complications. ${ }^{8}$ Diabetic patients tend to exhibit signs of anxiety and poor emotional health due to negative diabetes perception, loss of control, and fear of complications. ${ }^{9,10}$ A "response shift", which is an internal change of values, standards, and concepts regarding QoL must then take place in order for the patient to be able to confront the chronic disease and all that it entails. ${ }^{11}$

In patients suffering from DM, particularities in QoL perception arise due to the strain of the chronic nature of the disease and to the multitude of complications that can derive from it. Cardiovascular complications such as atherosclerosis, hypertension, and cardiac autonomic neuropathy are also found in diabetes and represent a permanent stressor for the diabetic patient. Chronic kidney disease (CKD) has diabetes as a main cause in its final stages, leading to dialysis. Retinopathy is yet another complication present, one that can lead to visual impairment or even blindness. Diabetic neuropathy (DN) can affect the peripheral nerves, leading to foot ulcerations and even amputations, or autonomic ones, causing gastrointestinal, urinary and sexual dysfunctions. There are studies that confirm the link between depression and $\mathrm{DN}$, even more, the link between the level of depression and the severity of the neuropathy. Depression is shown to appear shortly after the debut of diabetic complications, especially peripheral neuropathy. ${ }^{5,9,10}$

Several studies, including here randomized clinical trials, described the influence of several factors that had an impact on the QoL in cohorts of patients diagnosed with both type 2 diabetes mellitus (T2DM) ${ }^{12,13}$ and type 1 diabetes mellitus (T1DM) ${ }^{14}$ however, there were observed significant differences regarding the importance and impact on QoL of different factors, differences generated mainly by regional, geographical, and cultural differences between the studied cohorts. Emphasized by this fact and considering that the Romanian patients have a set of cultural and social particularities, we considered that studying these factors and their impact on Romanian patients' QoL is of paramount importance.

Having taken all of the above into consideration, the aim of this study is to evaluate which factors may influence the perception on the QoL in Romanian patients with T2DM.

\section{Patients and methods Study design and patients}

In this cross-sectional, noninterventional study, a cohort of 198 patients with T2DM was enrolled according to a consecutive-case population-based method. All patients were attending scheduled monitoring visits at the outpatient of the Diabetes Clinic from the Emergency Hospital in Timisoara and were previously diagnosed with T2DM. At the time of the screening, the following were considered exclusion criteria: the inability to provide informed consent, inability to provide accurate anamnestic medical history data, prior history of nondiabetic neuropathies, major cardiovascular events 3 months prior to screening, or any other condition that, in the investigators' opinion, could lead to biases in the study results.

The studied group characteristics were representative of the Romanian T2DM population; the patients enrolled in the study had a median age of 61 years, a median diabetes duration of 7 years, and $55.1 \%$ of them $(n=109)$ being females.

The study design, protocol, and informed consent form were reviewed and approved by the Ethics Committee of the Emergency Hospital Timisoara (no 328/2014); all patients provided written informed consent prior to any study procedure or activity.

\section{QoL assessment}

The QoL was investigated using the quality of life index diabetes version III (QoL) proposed by Ferrans and Powers, which is a validated instrument for the diabetic population and which was validated for the Romanian population. ${ }^{15}$ This instrument measures both satisfaction and importance of various aspects of life: health and functioning, social and economic, and psychological and family relationships. The instrument consists of two major parts: first in which satisfaction in previously mentioned items is measured and the second which measures the importance of these items for the questioned person. A higher score is associated with an increased QoL. ${ }^{15}$

\section{Diabetes complications and comorbidities assessment}

DN was diagnosed using the Michigan Neuropathy Screening Instrument (MNSI). This is a validated score instrument for $\mathrm{DN}$, being widely used for positive diagnosis and severity measurement of neuropathy. It consists of two distinct parts: a 15-item questionnaire that assesses the patient's signs and symptoms regarding the neuropathy and a lower extremity examination performed by the health care professional 
in which pathological alterations are scored. The positive diagnosis of neuropathy was established if a questionnaire score higher than 7 or a clinical examination score higher than 2.5 was found. At the same time, a higher MNSI score was associated with a more severe neuropathy.

CKD was diagnosed according to Kidney Disease Improving Global Outcomes 2012 criteria and the diagnosis of retinopathy based on the funduscopic examination results performed by an ophthalmologist specialized in the eye's diseases of diabetic patients. ${ }^{16}$ The presence of cardiac autonomic neuropathy was investigated using the measurement of changes in postural blood pressure. A positive diagnosis for postural hypotension was defined by a drop in systolic blood pressure of $20 \mathrm{mmHg}$ or diastolic blood pressure of $10 \mathrm{mmHg}$ within 3 minutes after changing the body position from supine to standing. ${ }^{17}$

\section{Clinical, anthropometric, and laboratory data}

Data on the patient's age, diabetes duration, and smoking status were collected from the patient's medical records. The HbA1c level was measured using an NGSP-standardized and DCCT-compliant immune-turbidimetric assay (Roche), having an intermeasurement coefficient of variation of $1.64 \%$ according to the manufacturer's specifications. Body mass index (BMI) was calculated by dividing the patient's weight (measured in kilograms) to the square of the patient's height (measured in meters).

\section{Depression and quality of diabetes- related self-care activities}

To evaluate the presence and the severity of depression, we used the Patient's Health Questionnaire-9 (PHQ-9). With its 9 items, the PHQ-9 test consists of the actual 9 criteria on which the diagnosis of DSM-IV depressive disorders is based. The PHO-9 is a dual-purpose instrument that can establish, with the same 9 items, depressive distress and grade depressive symptom severity. A higher PHQ-9 score is associated with more severe depression. ${ }^{18}$

The quality of diabetes-related self-care activities was evaluated using the Summary of Diabetes-related Self-Care Activities questionnaire (SDSCA), which is a validated tool in the population of DM patients. A higher SDSCA score is associated with a better disease self-care in these patients. The SDSCA questionnaire includes items assessing the following aspects of diabetes care: general and specific diet measures, exercise, blood-glucose testing, foot care, and smoking. ${ }^{19}$

\section{Statistical analysis}

Data were collected and analyzed using the Statistical Package for the Social Sciences (SPSS) v.17 software suite (SPSS Inc., Chicago, IL, USA) and are presented as medians and interquartile range for continuous variables without Gaussian distribution, average \pm standard deviation for continuous variables with Gaussian distribution or number of individuals and percentage from the subgroup total in case of nominal variables. The normality of the variable's distribution was tested prior to analysis according to the following methods: Kolmogorov and Smirnov normality test used in parallel with Shapiro-Wilk and normality plot analysis (at these tests a $P$-value $<0.05$ was considered to be associated with a nonGaussian values distribution).

To assess the significance of the differences between groups, Mann-Whitney $U$, Kruskal-Wallis (medians, nonGaussian populations), and chi-square (percentages) tests were used. The correlation between studied variables was evaluated using Spearman's rank sum correlation coefficient (non-Gaussian distributed variables), its statistical significance being assessed using the $t$-distribution score test.

The individual impact of several confounding factors on the variance of a continuous variable was assessed by building multivariate regression models. The predictors, in the final regression equations, were accepted according to a repeated backward-stepwise algorithm (inclusion criteria $P<0.05$ and exclusion criteria $P>0.20$ ) in order to obtain the most appropriate theoretical model to fit the collected data. The quality of the model was described using the accuracy of the prediction by adjusted $R^{2}$ (multivariate linear regression). In this study, a $P$-value of $<0.05$ was considered as the threshold for statistical significance.

\section{Results \\ Demographic, social, psychological, and anthropometric factors}

In our study group, the perception on the QoL was associated with neither the patient's age (Spearman's $r=0.016 ; P=0.820$ ) nor the duration of DM (Spearman's $r=-0.004 ; P=0.957$ ). There were no differences regarding the QoL's perception with respect to gender ( $P=0.343)$, smoking status ( $P=0.595)$, or dangerous alcohol consumption habits, as evaluated with the AUDIT-C questionnaire $(P=0.775)$.

Our results are pointing to a significant negative correlation between the patients' BMI and their perception on the QoL (Spearman's $r=-0.158 ; P=0.026$ ), indicating thus that patients with a higher BMI tend to have a decreased QoL (Figure 1). 


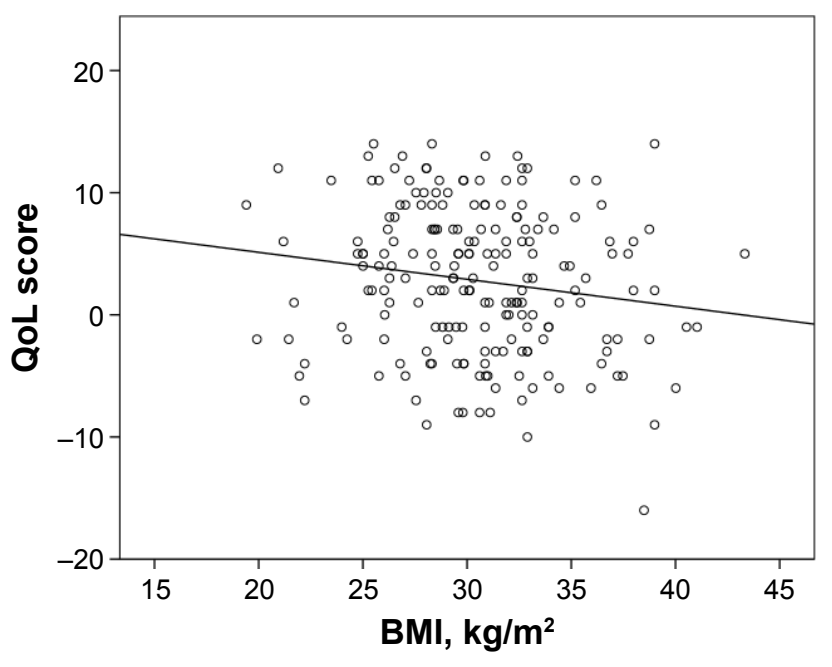

Figure I The correlation between the BMI and the patient's QoL. Abbreviations: BMI, body mass index; QoL, quality of life.

At the same time, the presence and severity of depression, assessed using the PHQ-9 score, was reverse and significantly correlated with the QoL (Spearman's $r=-0.345 ; P<0.001$ ), denoting that patients with more severe depression symptomatology are having a decreased QoL (Figure 2).

The details regarding the impact of the demographic, social, psychological, and anthropometric factors on the patient's QoL are summarized in Table 1.

\section{Diabetes-related factors influencing the QoL}

Glycemic control and patient's adherence to treatment

The QoL was not significantly correlated with the quality of the glycemic control measured using the HbAlc

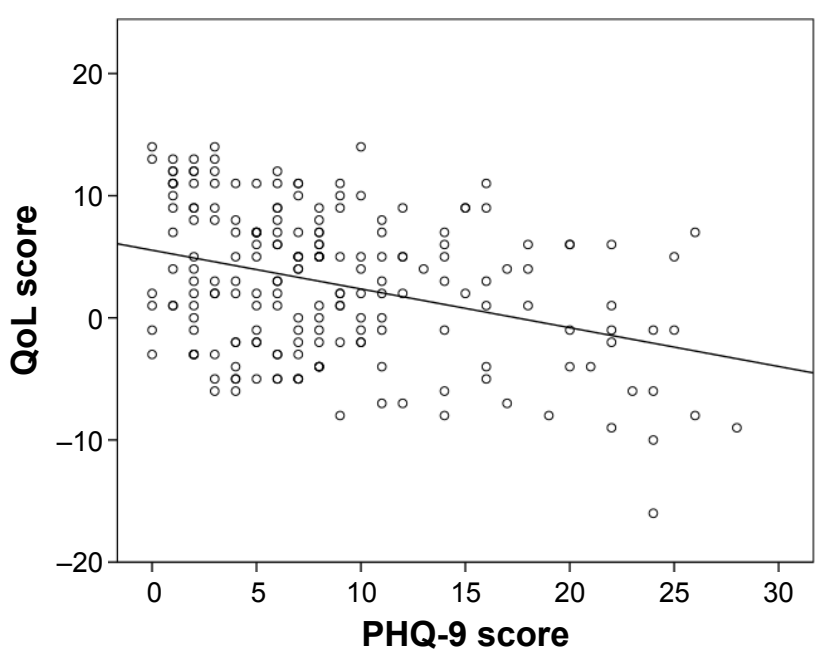

Figure 2 The association between depression and perception on the QOL. Abbreviations: PHQ-9, Patient's Health Questionnaire-9; QoL, quality of life.
Table I Association between demographic, social, psychological, and anthropometric factors and patient's QoL

\begin{tabular}{|c|c|c|}
\hline Parameter & Patient's QoL & $P$-value \\
\hline Patient's age (years) ${ }^{\mathrm{a}}$ & 0.016 & 0.820 \\
\hline DM duration (years) ${ }^{\mathrm{a}}$ & -0.004 & 0.957 \\
\hline BMI $\left(\mathrm{kg} / \mathrm{m}^{2}\right)^{\mathrm{a}}$ & -0.158 & 0.026 \\
\hline PHQ-9 score (points) & -0.345 & $<0.001$ \\
\hline Males $^{b}$ & $2(10)$ & 0.775 \\
\hline Females $^{\mathrm{b}}$ & $4(9)$ & \\
\hline Nonsmokers ${ }^{\mathrm{b}}$ & $3(10)$ & 0.595 \\
\hline Smokers ${ }^{\mathrm{b}}$ & $4(9)$ & \\
\hline
\end{tabular}

Notes: aResults are presented as Spearman's correlation coefficient. 'Results are presented as median and (interquartile range).

Abbreviations: BMI, body mass index; DM, diabetes mellitus; PHQ-9, Patient's Health Questionnaire-9; QoL, quality of life.

value (Spearman's $r=-0.114 ; P=0.110$ ); in contrast, it was positively and significantly correlated with the patient's adherence to diabetes-related self-care activities (Spearman's $r=0.338 ; P<0.001$; Figure 3 ).

\section{Diabetes complications and comorbidities}

The study results are pointing to a decreased QoL in patients having diabetes complications. This decrease was observed for patients with neuropathy ( -1 vs 5 points, $P<0.001)$, CKD ( -1 vs 4 points, $P<0.001$, Figure 4 ), retinopathy ( 1 vs 5 points, $P<0.001)$, and cardiac autonomic neuropathy $(-1$ vs 4 points, $P=0.001$ ).

Moreover, the severity of neuropathy, assessed using the MNSI score was reversed and significantly correlated with the patient's QoL (Spearman's $r=-0.483, P<0.001$, Figure 5).

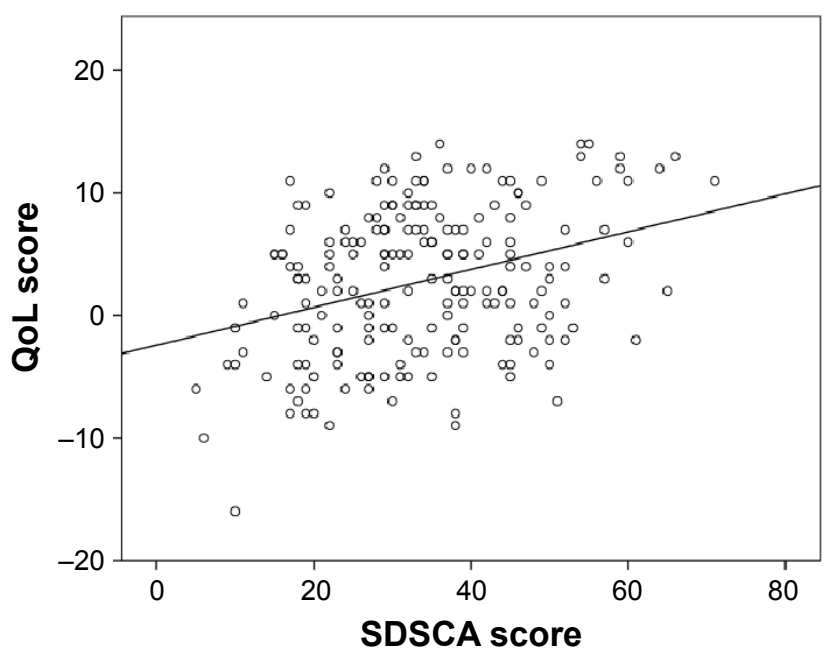

Figure 3 The correlation between the quality of diabetes-related self-care activities and patient's QoL.

Abbreviations: QoL, quality of life; SDSCA, Summary of Diabetes-related SelfCare Activities. 


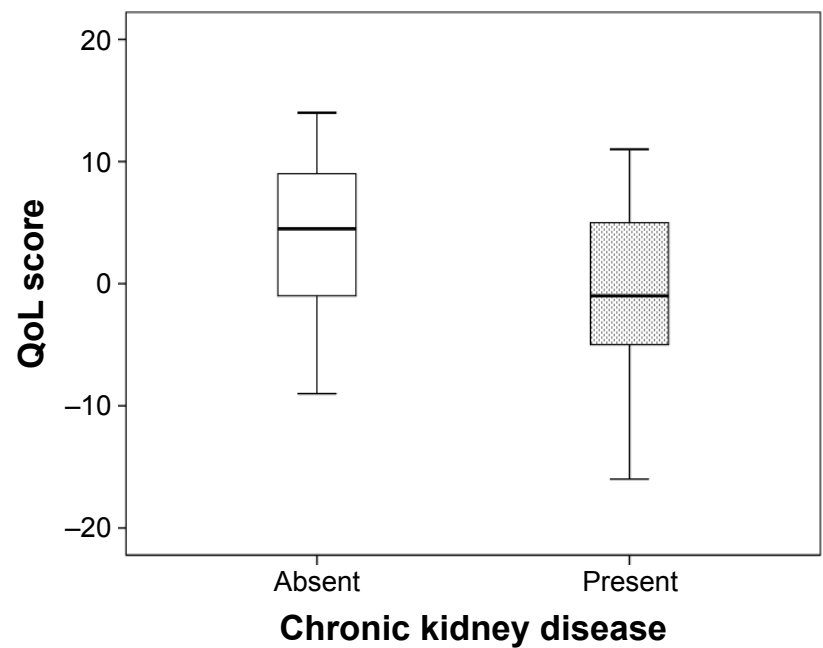

Figure 4 Comparison of QoL in patients with vs without neuropathy. Abbreviation: QoL, quality of life.

The presence of hypertension as a comorbidity of diabetes seemed to not influence significantly the patient's QoL.

An analysis of diabetes complications and comorbidities impact on the patient's perception regarding the QoL is presented in Table 2.

\section{Multivariate regression analysis}

Since the QoL in patients with DM may be influenced by multiple factors, factors between which and independent relation may exist, we aimed to evaluate which factors are possible independent predictors and which are only cofactors in influencing the perception on the QoL in this cohort of patients. To evaluate the independent impact for each evaluated factor we built, with the collected parameters, a

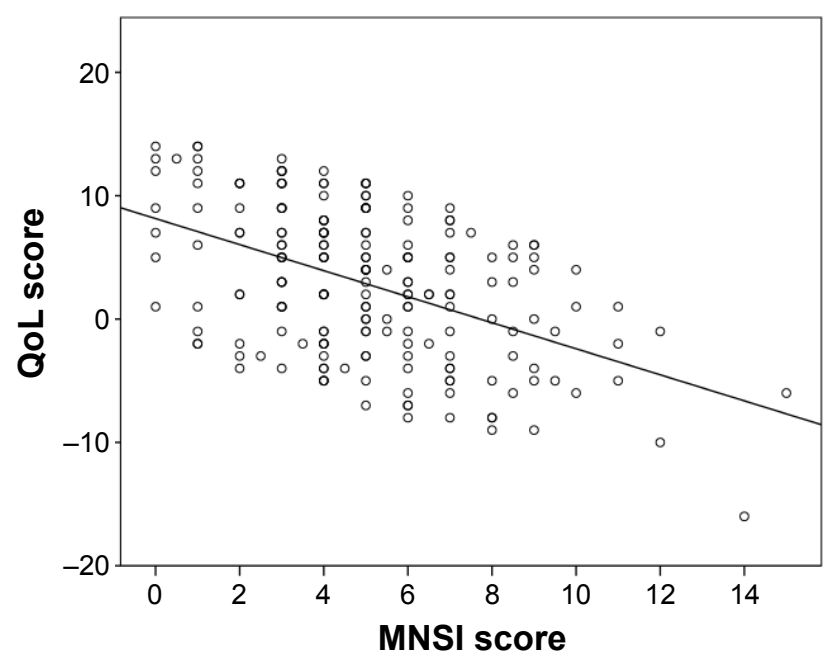

Figure 5 Correlation between the neuropathy's severity and patient's QoL. Abbreviations: MNSI, Michigan Neuropathy Screening Instrument; QoL, quality of life.
Table 2 Diabetes complications and comorbidities impact on patient's QoL

\begin{tabular}{|c|c|c|}
\hline $\begin{array}{l}\text { Complications and } \\
\text { comorbidities }\end{array}$ & Patient's QoL & $P$-value \\
\hline Diabetic neuropathy & & $<0.001$ \\
\hline Absent & $5(8)$ & \\
\hline Present & $-1(8)$ & \\
\hline Chronic kidney disease & & $<0.001$ \\
\hline Absent & $4(10)$ & \\
\hline Present & $-I(10)$ & \\
\hline Retinopathy & & $<0.001$ \\
\hline Absent & $5(10)$ & \\
\hline Present & I (9) & \\
\hline Cardiac autonomic neuropathy & & 0.001 \\
\hline Absent & $4(9)$ & \\
\hline Present & $-1(8)$ & \\
\hline Hypertension & & 0.587 \\
\hline Absent & $2(9)$ & \\
\hline Present & $4(10)$ & \\
\hline
\end{tabular}

Note: Results are presented as median and (interquartile range).

Abbreviation: QoL, quality of life.

multivariate regression model having as outcome variable the value of QoL. To obtain the best fit model, the initial collected parameters (patient's age, diabetes duration, gender, HbA1c, MNSI score, smoking status, alcohol consumption, BMI, PHQ-9 score, SDSCA score, and the presence of complications and comorbidities: CKD, retinopathy, and hypertension) were added in the regression model according to a backward-stepwise criterion (inclusion criteria: $P<0.05$, exclusion criteria: $P>0.20$ ). On the basis of this inclusion algorithm, the following predictors were accepted in the final model: patient's age, the value of HbA1c, MNSI score, depression's severity (PHQ-9 score), the presence of retinopathy, and CKD.

According to our model, an independent significant impact on the patient's QoL had the severity of neuropathy (MNSI score), the presence of CKD and retinopathy, and the severity of depression. Our results are pointing that for each increase with a standard deviation in the value of the MNSI score, the depression score decreased with 0.356 standard deviations $(\operatorname{Exp}(\beta)=-0.356, P<0.001)$. In parallel, significant independent predictors were the $\operatorname{HbA} 1 \mathrm{c}$ value $(\operatorname{Exp}(\beta)=-0.103$; $P=0.010)$, the presence of $\operatorname{CKD}(\operatorname{Exp}(\beta)=-0.120 ; P=0.009)$, and diabetic retinopathy $(\operatorname{Exp}(\beta)=-0.113 ; P=0.013)$. The other analyzed predictors had no significant independent impact on the patient's QoL.

\section{Discussion}

The study results show a decreased QoL in patients suffering from diabetic complications, such as neuropathy, CKD, retinopathy, and cardiac autonomic neuropathy. The severity 
of neuropathy assessed using the MNSI score was reversed and significantly correlated with the patient's QoL. In the case of diabetic foot ulcer (DFU) presence or history, physical impairment gravely affected QoL, ${ }^{20}$ to such an extent that mobile amputees had a better psychological status than DFU patients. ${ }^{21}$ The proliferative diabetic retinopathy diagnosis increases depression and negative life experiences for a period of 2 years after the diagnosis, as proven by several studies. ${ }^{22,23}$

Another factor that was positively and significantly correlated with QoL was the patient's adherence to diabetes-related self-care activities, such as proper diet, exercise routine, and glycemic control. These elements play a crucial role in preventing the apparition of complications and in maintaining a healthy BMI, as patients with a higher BMI have been found to have a decreased QoL, underlining the significant negative correlation between the two. It has been found that if two or more diabetes-related complications were present, there was a significantly associated likelihood that patients with diabetes showed clinical symptoms of depression or anxiety. ${ }^{24}$ Signs of depression and emotional distress should be closely monitored, as they influence self-care behaviors in a negative manner, studies have shown. ${ }^{25,26}$

When referring to the association between QoL in patients with diabetes and treatment regimen, it was found that the QoL decreases as the treatment regimen advances, being perceived as best when they only had a diet and exercise program, having less diabetes-related worries than patients taking oral medication, whereas those taking insulin reported more burden of illness and less treatment satisfaction than those controlling their glucose levels orally or by diet and exercise alone. ${ }^{27}$

The strength of this study lies in the fact that it is the only one that addresses the QoL in diabetic patients in Romania. Although such studies exist elsewhere, we must keep in mind the particularities of the Romanian population in what culture, tradition, education, health, and socioeconomic status are regarded. The results of this study are based on a large patient cohort, one that very well comprises the characteristics of the diabetic population in Romania, thus being a very representative pool.

The limitative character of this study is rendered by the transversal manner in which the evaluation of glycemic control was made: altering of QoL in respect to glycemic control might necessitate a longer timespan than 3 months, which is the period comprised by our analyzing of HbA1c.

The number of complications has also been reported as a strong predictor of QoL in a population of diabetic patients by Trief et al, ${ }^{28}$ while Jacobson et al noted that the greater the severity of complications, the lower scores on all QoL scales,${ }^{27}$ these results being in accord with our findings.

The authors' findings are mixed regarding the relation between the QoL and duration of diabetes. While several studies have found that a decreased QoL was associated with an increased duration of diabetes, ${ }^{29}$ others as ourselves have found no relevant association between the two. Peyrot and Rubin found no notable association between diabetes duration and depression in those with both T1DM and T2DM, ${ }^{24}$ nor did a Swedish study on type 2 diabetes patients, whose evaluations were done using scores on Bradley's well-being or treatment satisfaction questionnaires. ${ }^{30}$

QoL is an extremely important component in the management of any disease, especially chronic ones such as diabetes. The diabetic patient's QoL is influenced by a series of factors, such as neuropathy, CKD, retinopathy, cardiac autonomic neuropathy, and BMI. By identifying this series and by managing it, we could improve the QoL of our patients. Improvements in QoL should always be at the center of the patient's treatment and global management.

\section{Conclusion}

In patients with T2DM, the perception on the QoL is significantly influenced by the presence and severity of associated complications (neuropathy, CKD, and retinopathy), by the BMI, and by the presence and severity of depression. Given the modifiable character of these factors, special care should be provided in order to improve QoL in patients with T2DM. Also of great importance are the study's results that demonstrated that patients with decreased QoL simultaneously present a decrease in what the quality of diabetes-related self-care activities is concerned, thus leading to a decreased overall diabetes prognosis and an increase in the incidence of diabetes-associated complications.

\section{Acknowledgment}

This research was supported by an internal grant, funded by "Victor Babes" University of Medicine and Pharmacy, Timisoara, Romania (PLURINEURODIAB. PII-C4-TC2016-16441-01).

\section{Disclosure}

The authors report no conflicts of interest in this work.

\section{References}

1. World Health Organization. The World Health Organization Quality of Life Assessment (WHOQOL): position paper from the World Health Organization. Soc Sci Med. 2005;41(10):1403-1409. 
2. George W. Torrance Utility approach to measuring health-related quality of life. J Chronic Dis. 1987;40(6):593-603.

3. Ferrans CE. Definitions and conceptual models of quality of life. In: Lipscomb J, Gotay CC, Snyder C, editors. Outcomes Assessment in Cancer. Cambridge, England: Cambridge University; 2005:14-30.

4. Guyatt G, Feeny DH, Patrick D. Measuring health-related quality of life. Ann Intern Med. 1993;118(8):622-629.

5. American Diabetes Association. Diagnosis and classification of diabetes mellitus. Diabetes Care. 2004;27(Suppl 1):s5-s10.

6. World Health Organization. Global Report on Diabetes. Geneva, Switzerland: WHO; 2016. Available from: http://apps.who.int/iris/bits tream/10665/204871/1/9789241565257_eng.pdf. Accessed August 30, 2016.

7. Wild S, Roglic G, Green A, Sicree R, King H. Global prevalence of diabetes: estimates for the year 2000 and projections for 2030. Diabetes Care. 2004;27(5):1047-1053.

8. American Diabetes Association. Diagnosis and classification of diabetes mellitus. Diabetes Care. 2012;35(Suppl 1):S64-S71.

9. Balcıŏlu AS, Müderrisoğlu H. Diabetes and cardiac autonomic neuropathy: clinical manifestations, cardiovascular consequences, diagnosis and treatment. World J Diabetes. 2015;6(1):80-91.

10. Rekleiti M, Sarafis P, Saridi M, et al. Investigation of depression in Greek patients with diabetic peripheral neuropathy. Glob J Health Sci. 2013;5(5):107-114.

11. Sprangers MA, Schwartz CE. Integrating response shift into healthrelated quality of life research: a theoretical model. Soc Sci Med. 1999;48(11):1507-1515.

12. Johari N, Manaf ZA, Ibrahim N, Shahar S, Mustafa N. Predictors of quality of life among hospitalized geriatric patients with diabetes mellitus upon discharge. Clin Interv Aging. 2016;11:1455-1461.

13. Bannier K, Lichtenauer M, Franz M, et al. Impact of diabetes mellitus and its complications: survival and quality-of-life in critically ill patients. J Diabetes Complications. 2015;29(8):1130-1135.

14. Bakker SF, Pouwer F, Tushuizen ME, Hoogma RP, Mulder CJ, Simsek S. Compromised quality of life in patients with both Type 1 diabetes mellitus and coeliac disease. Diabet Med. 2013;30(7): 835-839.

15. Ferrans CE, Powers MJ. Quality of life index: development and psychometric properties. ANS Adv Nurs Sci. 1985;8(1):15-24.

16. Andrassy KM; CKD Work Group. Kidney disease: improving global outcomes (KDIGO). KDIGO 2012 clinical practice guideline for the evaluation and management of chronic kidney disease. Kidney Int. 2013; 86(Suppl 3):1-150.
17. Herman WH, Pop-Busui R, Braffett BH, et al. Use of the Michigan neuropathy screening instrument as a measure of distal symmetrical peripheral neuropathy in type 1 diabetes: results from the diabetes control and complications trial/epidemiology of diabetes interventions and complications. Diabet Med. 2012;29(7):937-944.

18. Kroenke K, Spitzer RL, Williams JBW. The PHQ-9: validity of a brief depression severity measure. J Gen Intern Med. 2001;16(9):606-613.

19. Toobert DJ, Hampson SE, Glasgow RE. The summary of diabetes self-care activities measure. Diabetes Care. 2000;23(7):943-950.

20. Meijer JW, Trip J, Jaegers SM, et al. Quality of life in patients with diabetic foot ulcers. Disabil Rehabil. 2001;23(8):336-340.

21. McNeely MJ, Boyko EJ, Ahroni JH, et al. The independent contributions of diabetic neuropathy and vasculopathy in foot ulceration. Diabetes Care. 1995;18(2):216-219.

22. Wuslin LR, Jacobson AM, Rand LI. Psychosocial aspects of diabetic retinopathy. Diabetes Care. 1987;10(3):367-373.

23. Wuslin LR, Jacobson AM. Visual and psychological function in PDR (Abstract). Diabetes. 1989;38(Suppl 1):242A.

24. Peyrot M, Rubin RR. Levels and risks of depression and anxiety symptomatology among diabetic adults. Diabetes Care. 1997;20(4): $585-590$.

25. Piette JD, Richardson C, Valenstein M. Addressing the needs of patients with multiple chronic illnesses: the case of diabetes and depression. Am J Manag Care. 2004;10(2 Pt 2):152-162.

26. Peyrot M, McMurry JF Jr, Kruger DF. A biopsychosocial model of glycemic control in diabetes: stress, coping and regimen adherence. J Health Soc Behav. 1999;40(2):141-158.

27. Jacobson AM, de Groot M, Samson JA. The evaluation of two measures of quality of life in patients with type I and type II diabetes. Diabetes Care. 1994;17(4):267-274.

28. Trief PM, Grant W, Elbert K, Weinstock RS. Family environment, glycemic control, and the psychosocial adaptation of adults with diabetes. Diabetes Care. 1998;21(2):241-245.

29. Aalto AM, Uutela A, Aro AR. Health related quality of life among insulin-dependent diabetics: disease-related and psychosocial correlates. Patient Educ Couns. 1997;30(3):215-225.

30. Wredling R, Stalhammar J, Adamson U, Berne C, Larsson Y, Oestman J. Well-being and treatment satisfaction in adults with diabetes: a Swedish population-based study. Qual Life Res. 1995;4(6):515-522.
Patient Preference and Adherence

\section{Publish your work in this journal}

Patient Preference and Adherence is an international, peer-reviewed, open access journal that focuses on the growing importance of patient preference and adherence throughout the therapeutic continuum. Patient satisfaction, acceptability, quality of life, compliance, persistence and their role in developing new therapeutic modalities and compounds to optimize

\section{Dovepress}

clinical outcomes for existing disease states are major areas of interest for the journal. This journal has been accepted for indexing on PubMed Central. The manuscript management system is completely online and includes a very quick and fair peer-review system, which is all easy to use. Visit http://www. dovepress.com/testimonials.php to read real quotes from published authors. 